\title{
Induction of specific macronuclear developmental mutations by microinjection of a cloned telomeric gene in Paramecium primaurelia
}

\author{
Eric Meyer \\ Laboratoire de Génétique Moléculaire, Ecole Normale Supérieure, 75230 Paris Cedex, France
}

\begin{abstract}
In Paramecium, the differentiation of a highly polyploid macronucleus from a diploid nucleus is accompanied by an extensive reorganization of the genome, involving reduction in chromosome size and formation of new telomeres at heterogeneous, but reproducible, positions. The results presented here, as well as work by others, indicate that telomere addition regions are not strictly determined by the micronuclear sequence, but are at least partially controlled by the old macronucleus. It is shown that microinjecting a high copy number of a plasmid containing the $G$ surface antigen gene into the macronucleus of wild-type cells specifically modifies the processing of the $G$ gene-bearing micronuclear chromosome at the following autogamy. Telomeric repeats are added upstream of the gene, rather than at their wild-type position $5 \mathrm{~kb}$ downstream of its 3 ' end, resulting in the deletion of the gene from the new macronucleus. This macronuclear mutation is unstable at the following autogamy, giving rise to many different telomere addition regions in different postautogamous clones. However, after several successive autogamies, cell lines can be obtained in which the telomeres reproducibly form in the same region. In crosses with wild-type cells, these macronuclear mutations show cytoplasmic inheritance; the micronuclei of the mutants are shown to be fully functional. The implications for the mechanism of choice of telomere addition sites are discussed.
\end{abstract}

Key Words: Developmental mutants; genome reorganization; macronuclear development; Paramecium primaurelia; telomere formation]

Received November 25, 1991; accepted December 23, 1991

Paramecium, like other ciliates, exhibits an interesting phenomenon of nuclear differentiation. Each cell contains two kinds of nuclei with markedly different structures and functions: The micronuclei are small, diploid nuclei that are apparently not transcribed but only serve to transmit genetic information from one sexual generation to the next through the processes of conjugation or autogamy; the macronucleus is a large, highly polyploid $(\sim 800 \mathrm{n})$ nucleus where most, if not all, of the cell's transcription takes place. During sexual events, the micronuclei undergo meiotic divisions to produce two identical haploid pronuclei, while the macronucleus breaks down into fragments that are diluted with successive fissions, and presumably degraded. In the case of conjugation, the two mating cells exchange one of their haploid pronuclei; fusion of the nuclei then gives two exconjugant cells with identical diploid zygotic nuclei. If only one mating type is present, the cell undergoes autogamy: The two haploid pronuclei fuse, giving rise to an entirely homozygous zygotic nucleus. In both cases, the zygotic nucleus then divides twice; two of the products remain micronuclei while the two others differentiate into two new macronuclei, which will segregate without division to the two daughter cells. Thus, autogamy produces two cells with independently developed macronuclei, which are called caryonides; conjugation produces four different caryonides, two from each exconjugant cell (for a complete description, see Sonneborn 1974).

The differentiation of a new somatic macronucleus from a diploid germinal nucleus is accompanied by an extensive and reproducible reorganization of the genome, involving amplification to the final ploidy level, chromosome fragmentation, and elimination of specific sequences. These processes occur to various extents in different species of ciliates (for review, see Yao 1989). In Paramecium primaurelia, reduction of chromosome size obviously occurs during macronuclear development, because the size of macronuclear chromosomes ranges from 50 to $800 \mathrm{~kb}$ (Caron and Meyer 1989), with an average of about $300 \mathrm{~kb}$ (Preer and Preer 1979), whereas the 30 to 60 micronuclear chromosome pairs (Sonneborn 1974), representing a haploid genome which has been variously estimated to contain between 45,000 and 280,000 kb (Gibson and Martin 1971; Cummings 1975; McTavish and Sommerville 1980), should be much longer on average. Because there appears to be little or no 
reduction in genome size during macronuclear development (Allen and Gibson 1972; McTavish and Sommerville 1980), chromosome breakage is very likely to occur, leading to the formation of new telomeres in the macronucleus.

Different classes of reproducible heterogeneity in the position of the telomeres added during macronuclear differentiation have been observed. The first class is exemplified by the macronuclear chromosomes harboring the $G$ surface antigen gene. Formal genetic studies have shown that this gene is present in a single copy in the micronuclear genome (Beale 1952), yet it is found on at least two different chromosomes in the macronucleus. One is $480 \mathrm{~kb}$ long, and the other is $250 \mathrm{~kb}$ long, with its restriction map identical with that of the last $250 \mathrm{~kb}$ of the 480-kb chromosome (Caron and Meyer 1989). The G gene lies near the telomere that is common to both chromosomes. A second class of heterogeneity can be defined, which involves smaller differences: Some chromosomes show multiple telomeres, 5-15 kb apart, at one of their ends. Such is the case of the chromosome bearing the A surface antigen gene of $P$. tetraurelia (Forney and Blackburn 1988). In contrast, the telomere located $\sim 5 \mathrm{~kb}$ downstream of the $\mathrm{G}$ gene of $P$. primaurelia is unique. The other ends of both the 250- and the 480-kb chromosomes, however, show the multiple telomere structure (F. Caron, unpubl.). Finally, a third class of heterogeneity is found within each of the telomeres, where the telomeric repeats are added at different nucleotides within a region spanning 200-800 bp (Baroin et al. 1987; Forney and Blackburn 1988). All three types of heterogeneity are found within caryonidal clones, that is, vegetative clones arising from a single macronuclear differentiation event. No conserved sequence element has been identified in the vicinity of telomeric repeat addition sites in any of the telomere addition regions studied. Furthermore, a mutant has been described in $P$. tetraurelia in which telomeres are added to sequences within the gene coding for the A surface antigen, rather than at their normal positions $8-26 \mathrm{~kb}$ from the $3^{\prime}$ end of the gene. This results in the deletion from the macronucleus of the $3^{\prime}$ part of the gene and downstream sequences (Forney and Blackburn 1987). Thus, there appears to be no specific telomere addition sequence. What then determines the position of telomere addition regions during macronuclear differentiation?

There is increasing evidence that the old macronucleus itself is playing an important role in the control of the genome reorganization. Some hereditary traits, such as the mating type in P. tetraurelia (Sonneborn 1977) or a trichocyst discharge mutation (Sonneborn and Schneller 1979), show cytoplasmic (non-Mendelian) inheritance in genetic crosses, due to the determination of the new macronucleus by cytoplasmic factors produced by the old macronucleus. The $\mathrm{d} 48$ mutation also shows cytoplasmic inheritance (Epstein and Forney 1984). The hereditary determinant of the mutation is located in the macronucleus, while the micronucleus is wild type (Harumoto 1986). In this case, it was shown that the old macronucleus determines the new one by specifying an alternative processing of the DNA during the developmental reorganization of the genome.

In this paper I show that an experimental modification of the genetic content of the macronucleus by microinjection of cloned DNA results in an altered processing of specific micronuclear chromosomal regions during the following genome reorganization. When DNA molecules from various origins are microinjected into the macronucleus of $P$. aurelia, they are processed to yield stable linear chromosomes bearing telomeric repeats $\left(\mathrm{C}_{4} \mathrm{~A}_{2}\right.$ and $\mathrm{C}_{3} \mathrm{~A}_{3}$ ) at their ends that can replicate autonomously during vegetative growth (Gilley et al. 1988), apparently without the need for a specific replication origin sequence. The injected DNA is lost at autogamy when the macronucleus is degraded and replaced by a new one derived from the micronucleus. When a plasmid containing the gene coding for surface antigen G of strain 156 (156G gene) of $P$. primaurelia is injected into the macronucleus of strain 168 cells, the $156 \mathrm{G}$ protein can be stably expressed at the cell surface, as detected by an immobilization test using specific antibodies (Caron and Meyer 1989|. After the following autogamy, the plasmidencoded protein rapidly disappears. Since autogamy usually does not affect serotype expression /autogamy of natural 156/168 heterozygotes expressing the G serotype, e.g., yields F2 clones expressing either the 156 or the 168 allele of the $G$ genel, the transformed cell lines should maintain the expression of the endogenous 168G gene. However, it was unexpectedly observed that the endogenous $168 \mathrm{G}$ protein, like the plasmid-encoded $156 \mathrm{G}$ protein, also disappears from the cell surface after autogamy of the transformed cell lines. This observation prompted an investigation of the structure of the new macronuclear genome, which showed that the disappearance of the $168 \mathrm{G}$ protein is due to the loss of the endogenous 168G gene from the new macronucleus. This work shows that transformation of wild-type cells with a high copy number of the G-gene plasmid results in an alternative telomerization pattern of the homologous micronuclear gene during the following macronuclear development.

\section{Results}

Induction of macronuclear mutations in strain 168

To see whether any change in the new macronuclear genome might be induced by the transformation, DNA was extracted from transformed cell lines before and after autogamy. Plasmid $\mathrm{pX} \Delta 4$ contains the entire transcribed sequence of the $156 \mathrm{G}$ gene, as well as $230 \mathrm{bp}$ of upstream sequences and $510 \mathrm{bp}$ of downstream sequences (see Fig. 1). The supercoiled form of this plasmid was injected into the macronucleus of 168 cells; DNA was extracted from the transformed cell lines and autoradiographs of Southern blots were scanned to estimate the amount of established plasmid. The copy number was approximately $4,000,24,000,21,600$, and 7,200 per macronucleus for clones $1,2,3$, and 4, respectively (Fig. $2 \mathrm{~A}, \mathrm{~B})$, that is, between 5 and 30 times the normal 

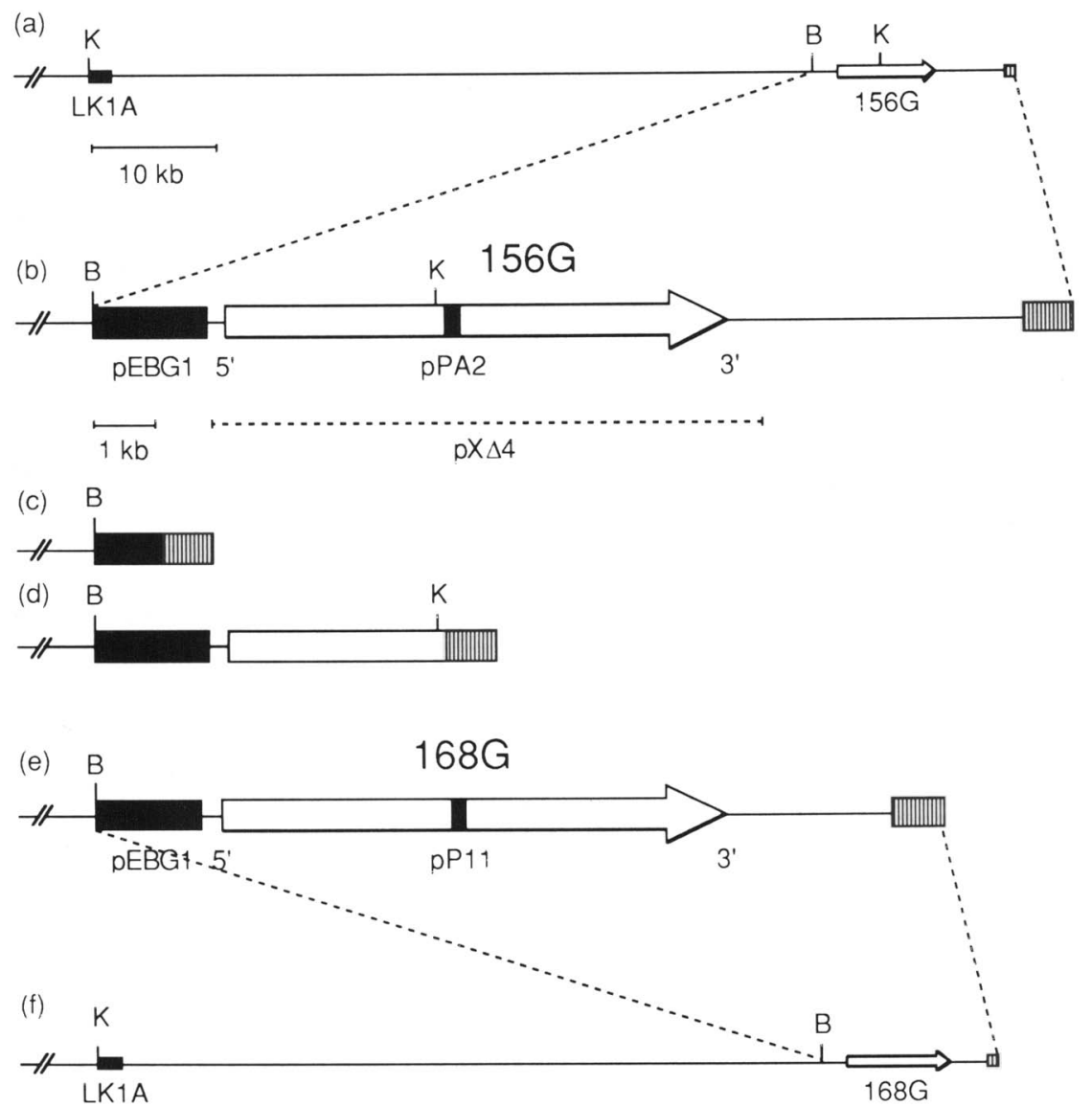

Figure 1. Map of the end of the wild-type $G$ gene bearing macronuclear chromosomes of strains $156(a, b)$ and $168(e, f) .(c)$ Map of the first-generation mutant telomere (after autogamy of the injected cell lines, both 156 and 168$)$. (d) Map of the s5 mutant telomere. Arrows represent the transcribed sequences of the genes. Hatched boxes represent the telomere addition regions. Black boxes represent the fragments used as probes for the Southern blots. The dotted line in $b$ indicates the sequence contained in plasmid $\mathrm{pX} \Delta 4$.

amount of the gene. Seven clones that were injected with a 1.6-kb linear EcoRI fragment derived from the upstream part of the $156 \mathrm{G}$ gene were included as controls (lanes 5-11). The 1.6-kb fragment did not replicate autonomously in the seven clones analyzed, as is shown by the absence of any hybridizing material at the appropriate position in Figure 2B. However, this fragment was integrated into the genomic DNA at a high copy number in clone 9 through nonhomologous recombination (data not shown; see Katinka and Bourgain 1992 for nonhomologous recombination of injected DNA), and possibly in some other clones at a lower copy number. All 11 injected clones were allowed to undergo autogamy, and one or two independent postautogamous caryonides were isolated for each clone and studied. Figure $2 \mathrm{C}$ shows a Southern blot of BamHI-digested DNA from both pre- and postautogamous cell lines. The presence of the $168 \mathrm{G}$ gene is revealed by hybridization with a 168 specific probe, pP11 (see Fig. 1). In wild-type cells, BamHI generates a $13.5-\mathrm{kb}$ telomeric fragment containing the $168 \mathrm{G}$ gene. This fragment is absent or much reduced in intensity in postautogamous clones derived from clones 2, 3, and 4. (Micronuclear DNA only represents $1 / 400$ of the total cellular DNA and is not detectable on the Southern blots.) None of the postautogamous clones derived from clones injected with the $1.6-\mathrm{kb}$ fragment (or from uninjected control cells, not shown) shows this defect, and neither does the post-autogamous clone derived from clone 1, which had a lower copy number of the plasmid. Hybridization of the same blot or Southern blots of EcoRI-digested DNA with various probes indicated that the entire coding sequence as well as the downstream sequences were missing from the macronucleus of the mutant postautogamous clones (not shown). Thus, transformation of 168 cells with a high copy number of plasmid pX $\Delta 4$ prevents the $168 \mathrm{G}$ micronuclear gene from being correctly processed into the new macronucleus after the following autogamy.

\section{Characterization of the deletions}

The postautogamous clones lacking the $168 \mathrm{G}$ gene were further analyzed to determine the extent of the deletion. A 1.9-kb KpnI-EcoRI fragment located $72 \mathrm{~kb}$ upstream lorientation is defined relatively to the orientation of the $G$ gene; see Fig. 1) from the telomere of the $G$ genebearing macronuclear chromosome was cloned from a jumping genomic library (F. Caron, in prep.). This fragment, LK1 A, was used to probe a pulse-field electrophoresis Southern blot of KpnI-digested DNA (Fig. 3A). The $72-\mathrm{kb}$ telomeric fragment generated by KpnI digestion in wild-type 168 cells and in the transformed cell lines is reduced in size to yield three main bands of about 60,52 , and $43 \mathrm{~kb}$. The relative intensities of these three bands 


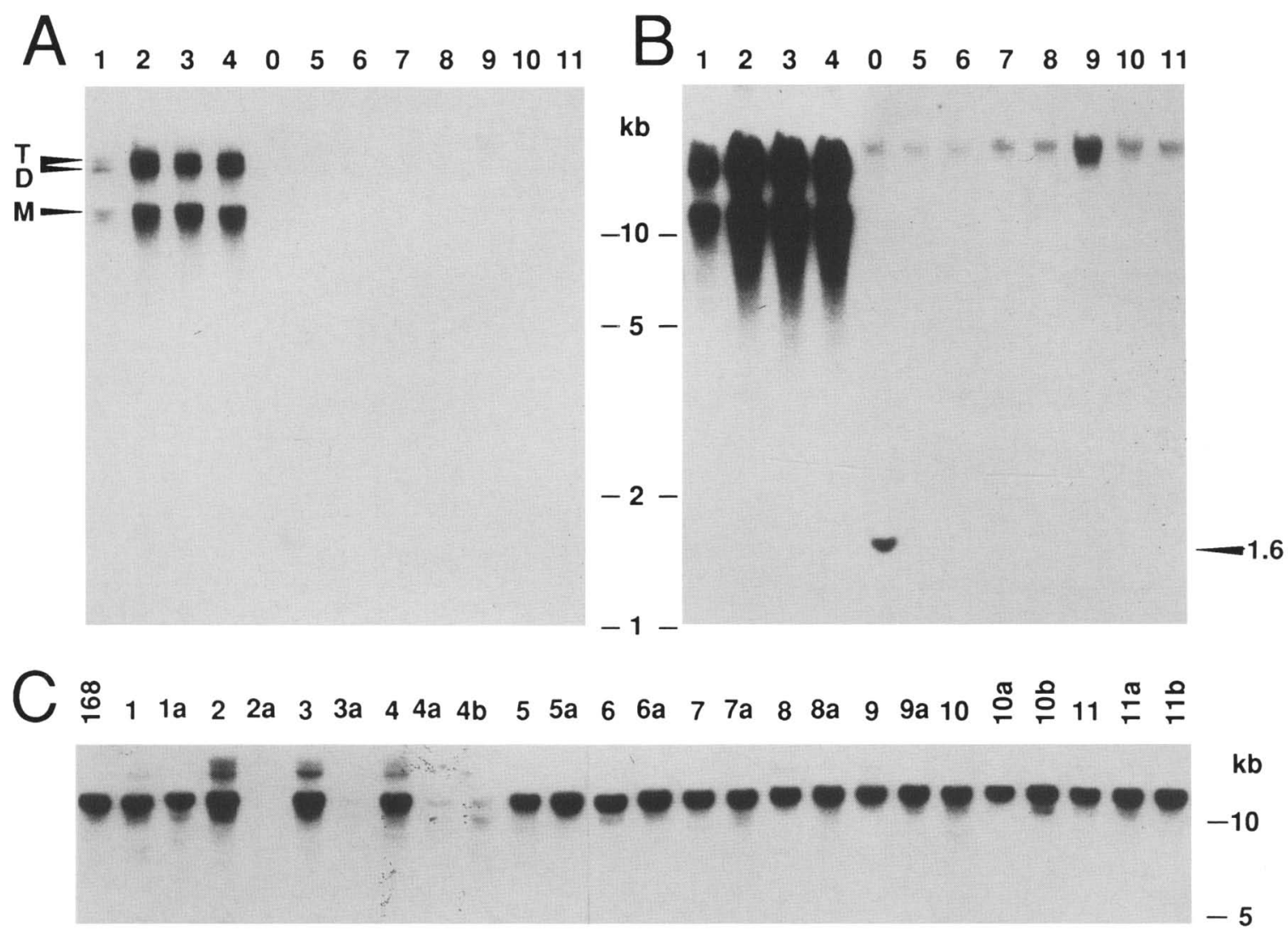

Figure 2. $(A, B)$ Southern blot of a $0.6 \%$ agarose gel electrophoresis of uncut total DNA extracted from 168 clones injected with plasmid pX $\Delta 4$ (lanes $1-4$ ) or with a $1.6-\mathrm{kb}$ fragment from the upstream part of the $156 \mathrm{G}$ gene (lanes $5-11$ ), probed with plasmid pX $\Delta 4$. Lane 0 contains uninjected 168 DNA mixed with $0.5 \mathrm{ng}$ of the $1.6-\mathrm{kb}$ fragment as a control. Exposure was $2.7 \mathrm{hr}(A)$ or $24 \mathrm{hr}(B)$. The arrows on the left indicate the position of the monomeric (M), dimeric (D), and trimeric $(T)$ forms of the injected plasmid. The signal seen with genomic DNA in clone 9 is more intense than the background cross-hybridization of plasmid pX $\Delta 4$ with the genomic $168 \mathrm{G}$ gene sequences (lane 0 ) because of integration of multiple copies of the $1.6-\mathrm{kb}$ fragment. (C) Southern blot of a $0.6 \%$ agarose gel electrophoresis of BamHI-digested DNA from the same injected cell lines and the postautogamous clones derived from them, probed with the 168-specific probe pPl1. Numbering of the clones is as in $A$ and $B$; the postautogamous clones are indicated by a or $b$. (Lane 168) Wild-type strain 168 DNA. The main band is the BamHI telomeric fragment containing the 168G gene (see Fig. 1). Faint bands visible above this band in clones $1,2,3$, and 4 are due to hybridization of the injected plasmid with pUC18 sequences contaminating the purified probe pPll.

vary among different postautogamous clones; the largest one, however, is always the most intense, except in clone la, where it is absent. Other minor bands are also seen below $43 \mathrm{~kb}$ in some clones. The simplest explanation of these results is that the G gene-bearing chromosomes suffer terminal deletions leading to the formation of new telomeres upstream from the wild-type telomere. If the $60-\mathrm{kb}$ band truly represents a telomeric fragment, it should show the characteristic size heterogeneity (heterogeneity in the exact point of addition of the telomeric repeats and in the number of repeats). The telomere corresponding to the $60 \cdot \mathrm{kb}$ band should be located a short distance downstream of the BamHI site, which is located $58.5 \mathrm{~kb}$ downstream of the KpnI site. Figure $3 \mathrm{C}$ shows a Southern blot of BamHI-digested DNA from the mutant postautogamous clones probed with a 1.9-kb fragment immediately downstream from the BamHI site (probe pEBG1; see Fig. 1). The expected smear is indeed seen between 1 and $2 \mathrm{~kb}$, together with a faint and variable band at $13.5 \mathrm{~kb}$, corresponding to a very small amount of wild-type telomeres (also seen in Fig. 2C). Thus, the 60$\mathrm{kb}$ band results from the addition of telomeres to the region located immediately upstream of the insert of plasmid $\mathrm{pX} \Delta 4$.

\section{Induction of macronuclear deletions in strain 156}

The transformation experiment was repeated with 156 recipient cells to see whether the overall sequence similarity between the injected plasmid and the endogenous gene had any effect on the phenomenon/the 156 and 168 sequences corresponding to the insert of pX $\Delta 4$ are only about $94 \%$ similar; Prat 1990). Injection of plasmid pX $\Delta 4$ into the macronucleus of 156 cells also resulted in the production of postautogamous mutants unable to express the G gene. A molecular analysis of three of these 


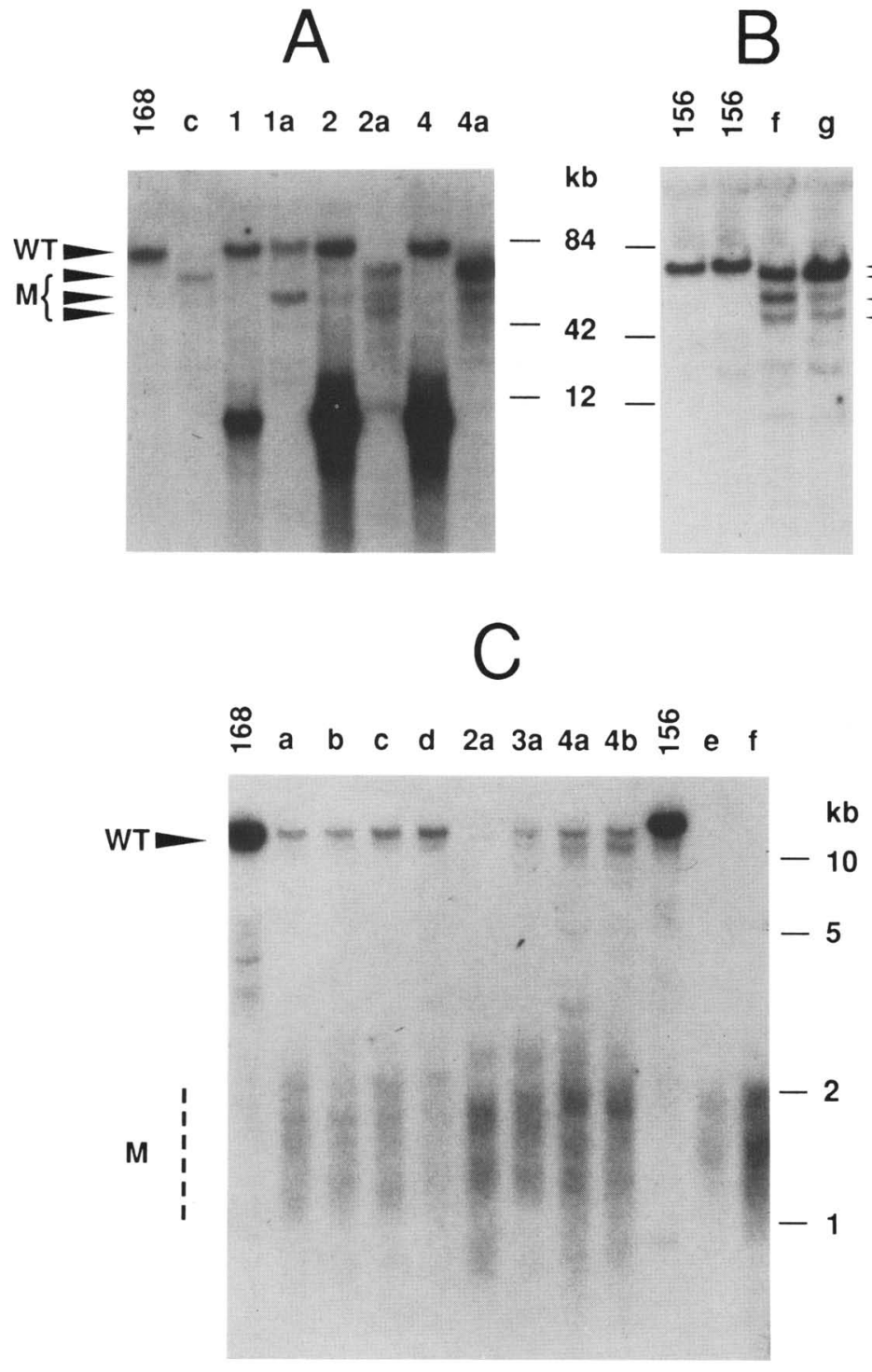

Figure 3. $(A, B)$ Southern blots of a pulse-field gel electrophoresis (CHEF) of KpnI-digested DNA from pre- and postautogamous 168 clones $(A)$ and postautogamous 156 clones $(B)$, probed with fragment LK1A. (C) Southern blot of a $0.6 \%$ agarose gel electrophoresis of BamHI-digested DNA from 168 and 156 postautogamous mutant cell lines, probed with fragment pEBGl. Numbering of the clones is as in Fig. 2. (Lanes $a, b, c, d$ ) Additional 168 mutants obtained after autogamy of a 168 clone injected with plasmid $\mathrm{pX} \Delta 4$ in a previous experiment. (Lanes $e, f, g$ ) 156 mutants obtained after autogamy of a 156 clone injected with pX $\Delta 4$. (Lane 168) Wild-type 168 DNA; (lane 156) wildtype 156 DNA. Arrowheads point to the position of wild-type (WT) or mutant main (M) telomeric fragments. The signal seen below $12 \mathrm{~kb}$ in lanes 1 , 2 , and 4 is due to hybridization of a KpnI fragment of the injected plasmid with the pUC18 moiety of the unpurified probe. The dotted line on the left of $C$ indicates the smear due to the mutant telomeres.

mutants is presented in Figure 3, B and C. Here again, probing a Southern blot of a pulse-field gel of KpnI-digested DNA with fragment LK1A showed the same set of bands as was seen with the 168 mutants (Fig. 3B). Note that the band observed in wild-type 156 DNA is not a telomeric fragment, because of the presence of a second $K p n I$ site in the coding sequence of the $156 \mathrm{G}$ gene (see Fig. 1). The reduction in size from this $64-\mathrm{kb}$ restriction fragment to the largest $(60-\mathrm{kb})$ telomeric fragment of the mutants is less obvious than in the case of the $168 \mathrm{mu}$ tants. To determine the origin of the minor bands of 52 and $43 \mathrm{~kb}$, a blot identical to the one shown in Figure 3B was probed with fragment $\mathrm{pEBGl}$; the same pattern was again observed (not shown). Since the $52-$ and $43-\mathrm{kb}$ fragments hybridize equally well with both probes LK1 A and pEBG1, which represent the two extremities of the $60-\mathrm{kb}$ mutant telomeric fragment, they cannot be due to larger terminal deletions, but rather represent additional dele- tions of internal sequences (between the KpnI site and the mutant telomere). Thus, in spite of the complex pattern seen with pulse-field electrophoresis, there is only one telomere addition region in the mutants. The additional internal deletions will not be discussed any further because $\{1\}$ their occurrence was unpredictable (sometimes they were not seen at all) and (2) they were no longer seen after a subsequent autogamy of the mutant cell lines, whereas terminal deletions were still observable (see section below, Stability of the macronuclear deletions). Figure $3 \mathrm{C}$ shows that the mutant telomere addition region spans exactly the same sequence in the 156 mutants as in the 168 mutants, between 1 and 2 $\mathrm{kb}$ downstream of the BamHI site. This implies a $14-\mathrm{kb}$ deletion instead of the $12-\mathrm{kb}$ deletion seen in $168 \mathrm{mu}$ tants, the 156 wild-type telomere being located $2 \mathrm{~kb}$ farther downstream than the 168 wild-type telomere (see Fig. 1). 


\section{Specificity of the deletions}

The only phenotype displayed by the mutants is their inability to express the $G$ serotype. Growth rate and morphology were indistinguishable from wild type. This argues against the idea of a generalized deletion of $12 \mathrm{~kb}$ or more from all macronuclear telomeres, which would probably affect essential genes, given the relatively small size of macronuclear telomeres. It is impossible to prove that no other telomere is being affected; however, three other macronuclear telomeres, for which probes were available, were analyzed by pulse-field electrophoresis and shown to have the same pattern in wild-type and mutant cells (data not shown). Two of these (including the other end of the $250 \mathrm{~kb}$ chromosome bearing the G gene) show multiple telomere addition regions $4-20 \mathrm{~kb}$ apart. The third one, like the $\mathrm{G}$ gene telomere, has a single telomere addition region. Thus, it appears that the deletion is specific for the $G$ gene telomere.

The deletion is also a specific effect of the $G$ gene plasmid. This is shown by a control experiment in which a different plasmid, YEpJB1-23-0 (Banroques et al. 1987), was injected into 156 cells. This plasmid is a yeast shuttle vector containing $2 \mu$ and yeast genomic sequences cloned into a pBR derivative; its size is similar to that of plasmid pX $\Delta 4(11.5 \mathrm{~kb})$. When the supercoiled form of this plasmid is injected into the macronucleus, the same pattern of replicating monomers and multimers is observed as following pX $\Delta 4$ injection (see Fig. 4A). Several cell lines transformed with this plasmid, with copy numbers similar or twice as high as the highest copy number of pX 44 in the first experiment (see Fig. 4A), were taken through autogamy. The processing of the micronuclear G gene was not affected: All postautogamous clones retained the ability to express the 156G antigen, and telomeres were added at the wild-type position in all the clones analyzed (an example is shown in Fig. 4B).

\section{Stability of the macronuclear deletions}

Two of the 156 mutants (e and f) were taken through a second autogamy to see whether the altered telomere position could have any influence on the processing of the $\mathrm{G}$ gene during the following macronuclear development. Figure 5 shows a Southern blot of BamHI-digested DNA probed with fragment pEBG1, which compares five independent postautogamous clones derived from $\mathrm{mu}-$ tant $e$ and 6 such clones from mutant $f$. The outcome of autogamy in these mutants was highly variable, but generally yielded a mix of mutant telomeres (resembling those of mutants e and f) and wild-type telomeres, with occasional multiple intermediate telomere positions. One clone $(\mathrm{f} 4)$ retained the same telomere distribution as mutant $f$ lautogamy was checked by staining three cells out of four after the first division of the caryonides to verify the presence of macronuclear fragments). Others $(\mathrm{f} 2, \mathrm{f} 3, \mathrm{f} 6, \mathrm{e} 2)$ had a sizable amount of wild-type telomeres and were actually expressing $G$ at the time of DNA extraction. Some $(\mathrm{e} 1, \mathrm{e} 3)$ only had telomeres at intermediate positions. None of the nine clones had a wild-type amount of wild-type telomeres.

\section{A B}

$\begin{array}{llll}12 & 13 & 14 & 2\end{array}$

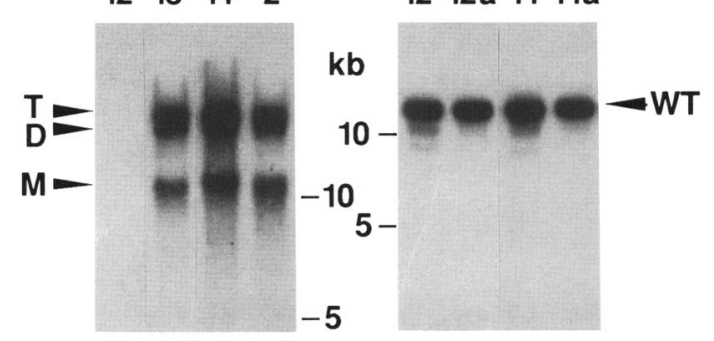

Figure 4. $(A)$ Southern blot of a $0.6 \%$ agarose gel electrophoresis of uncut total DNA extracted from 156 clones transformed with yeast plasmid YEpJB 1-23-0 (lanes 13,14), and from one 168 clone transformed with plasmid pX $\Delta 4$ (lane 2; this is clone 2 of Fig. 2), which did yield deletions of the endogenous $G$ gene after autogamy. Lane 12 is an uninjected control. The blot was probed with a 481-bp XmnI-BglI fragment of the ampicillinresistance gene, which is common to $\mathrm{pX} \Delta 4$ and YEpJB1-23-0, so that the copy numbers of the two plasmids can be compared directly. Scanning of an appropriate exposure indicated that the copy number of clone 13 is about the same as that of clone 2 , while that of clone 14 is twice as high. The arrowheads (left) indicate the position of the monomeric (M), dimeric (D), and trimeric $(T)$ forms of the injected plasmids. $(B)$ Southern blot of a $0.8 \%$ agarose gel electrophoresis of BamHI-digested DNA extracted from clones 12 and 14 and the postautogamous clones derived from them (lanes $12 a$ and $14 a$, respectively). The blot was probed with the $156 \mathrm{G}$-specific probe pPA2. The arrowhead indicates the wild-type BamHI telomeric fragment containing the G gene (WT).

One of these clones (e5) was then taken through successive autogamies. e5 had a grossly bimodal telomeric distribution, with two telomeric regions around 2 and 5 $\mathrm{kb}$ downstream of the BamHI site. Figure 6 shows that a single clone isolated after a third autogamy (s) retained only the telomere addition region around $5 \mathrm{~kb}$. Clone $\mathrm{s}$ was taken through a fourth autogamy: five independent postautogamous clones were analyzed (s1-s5), as well as a mass culture grown from 100 cells that were $97 \%$ autogamous $(\mathrm{sm})$. It is obvious from Figure 6 that the outcome of this last autogamy is much less variable than that of the autogamy of the mutants shown in Figure 5. All five independent postautogamous clones, as well as the mass autogamy of 100 cells, presented a similar telomeric distribution, slightly farther downstream than that of clone s. No wild-type telomeres were observed. Only s2 had a telomeric addition region significantly larger, extending 2-3 kb farther downstream. This variability is not significantly greater than the variability observed after the autogamy of strain 168, where the broadness of the telomeric distribution of different caryonides varies from 0.8 to $2 \mathrm{~kb}$, while the average position of telomeric repeats varies by more than $1 \mathrm{~kb}$ (A.-M. Keller, A. Le Mouël, F. Caron, M.D. Katinka, and E. Meyer, in prep.). 


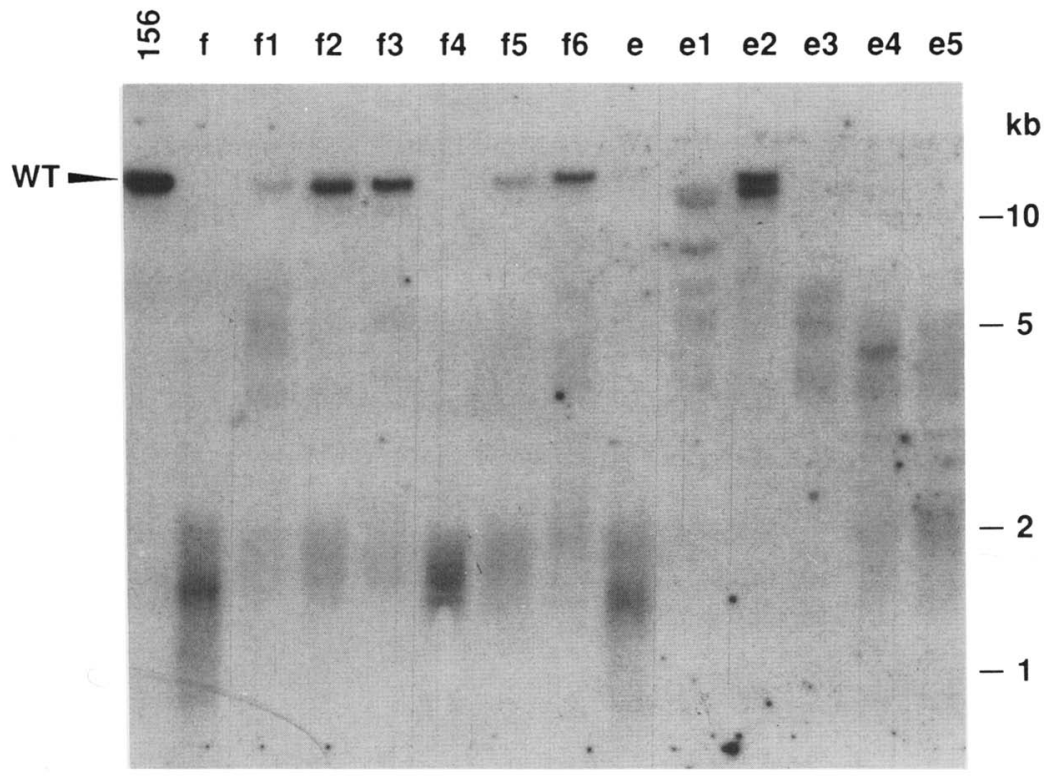

$\mathrm{kb}$

Figure 5. Southern blot of a $0.6 \%$ gel electrophoresis of BamHI-digested DNA from mutants e and $f$ and the postautogamous lines derived from them (lanes f1-f6,e1-e5), probed with fragment pEBGl. (Lane 156) Wild-type 156 DNA. The arrowhead indicates the wild-type telomeric fragment (WT).
Macronuclear DNAs from clones of the mutant cell lines after each autogamy (clones e5, s, s5, and the mass autogamy sm/ were also digested with $K p n I$ and analyzed on a Southern blot of a pulse-field gel probed with fragment LK1A to check for the presence of the minor 52and $43-\mathrm{kb}$ bands that were seen in the first-generation mutant $e$, as in mutants $f$ and $g$ (see Fig. 3B). Those bands were no longer seen in clone e5/after the second autog-

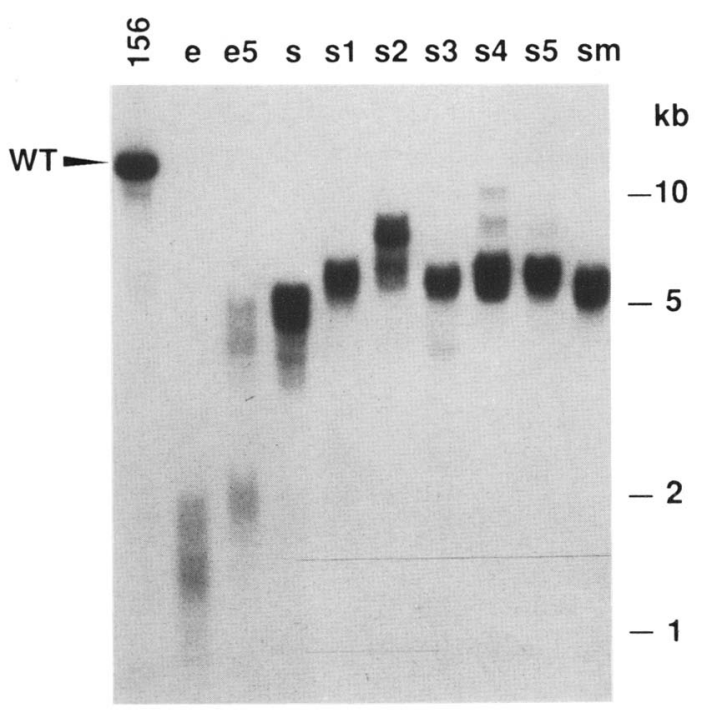

Figure 6. Southern blot of a $0.6 \%$ agarose gel electrophoresis of BamHI-digested DNA from cell lines obtained through successive autogamies of mutant $\mathrm{e}$, probed with fragment pEBGl. (Lane e5) Postautogamous clone derived from e (same as in Fig. 5); (lane s) postautogamous clone derived from e5; (lanes $s 1, s 2, s 3, s 4, s 5)$ five individual postautogamous clones derived from $s_{\text {; }}$ (lane $\mathrm{sm}$ ) culture grown from a mass autogamy of 100 cells of clone s; (lane 156) wild-type 156 DNA. The arrowhead indicates the wild-type telomeric fragment (WT). amy) nor in any of the following generations (data not shown|. Thus, the occasional internal deletions seen after autogamy of the injected cell lines, in contrast to the terminal deletions, did not reproduce in the new macronucleus after the following autogamy.

\section{Genetics of the macronuclear deletions}

To ensure that the micronuclear genome had not been modified by the microinjection or by the successive autogamies of the mutants, the ability of the micronuclear $\mathrm{G}$ gene to be processed correctly was tested by crossing the 156 mutant cell lines obtained after the fourth autogamy with wild-type 168 cells. If the micronucleus of the mutants is wild type, the $156 \mathrm{G}$ gene should be normally processed in the heterozygote progeny of the 168 parent. Mutant lines s2 and s5 were chosen because of the slight difference in their telomeric distribution. They were crossed with 168 cells expressing the G serotype (see Fig. 7 for a schematic representation of these crosses). The expression of both alleles was tested in each of the four caryonides after three or four divisions. Eight pairs were studied and showed expression of both alleles in the two caryonides derived from the 168 parent, while the two caryonides derived from the mutant expressed neither the 156 nor the 168 allele. Figure 8 shows a molecular analysis of two such crosses. DNA was extracted from the four caryonides, as well as from the parents just before the cross, digested with $B a m \mathrm{HI}$, and hybridized on a Southern blot successively with the 156-specific probe pPA2 (Fig. 8A) and the 168-specific probe pP11 (Fig. 8B). As expected from the expression pattern, both alleles have telomeres at the wild-type position in the caryonides derived from the 168 parent of both crosses. Thus, the micronucleus of the mutants contains copies of the $\mathrm{G}$ gene that can be correctly processed in a wild-type 


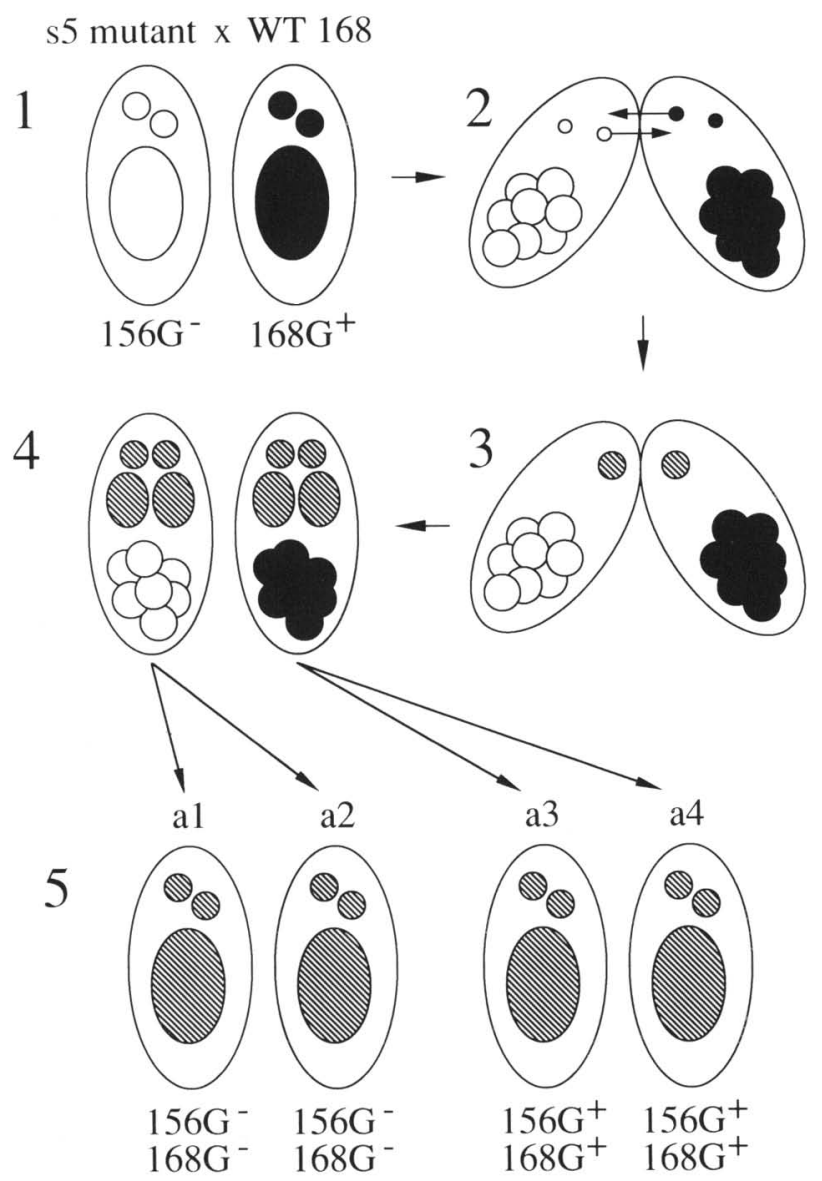

Figure 7. Schematic representation of the cross of the $156 \mathrm{mu}$ tant s5 with wild-type 168. (1) The large ovals represent the macronuclei and the circles represent the micronuclei. White and black symbolize the 156 and 168 genomes, respectively. The phenotypes indicated beneath the cells refer to the ability of the cells to express the two alleles of the G gene. (2) After pairing of the cells, the two micronuclei of each cell enter meiosis. Of the eight haploid products, seven degenerate and the remaining one divides once more to yield two identical haploid pronuclei, which are represented by small circles. The two mating cells then exchange one of their pronuclei. During this time the old macronucleus is fragmented. The fragments are represented clumped together for the sake of clarity. (3) Fusion of the exchanged pronucleus with the remaining one in each cell results in the formation of an identical heterozygous nucleus in the two mates. Heterozygous nuclei are depicted in gray. (4) The zygote nucleus then divides twice. Two of the products remain micronuclei, while the other two begin to differentiate into macronuclei (small ovals). (5) At the first division after conjugation, the two new macronuclei of each exconjugant segregate without division to the two daughter cells, called caryonides, while the two micronuclei divide by mitosis. The phenotype of the four caryonides obtained from this cross (a1, a2, a3, a4), corresponding to four independent differentiation events of identical micronuclei, illustrates the influence of the fragments of the old macronucleus on the differentiation process.

cell. In the caryonides derived from the mutant, the telomeres occupy multiple positions that are similar to the mutant or intermediate between the mutant and the wild-type positions. This is true for both the 156 and the 168 alleles. Thus, the wild-type micronuclear $168 \mathrm{G}$ gene is not correctly processed in the mutant cell. Therefore, it can be concluded that these mutations have a macronuclear pattern of inheritance. Two additional observations can be made from this experiment. First, the difference in telomeric distribution between mutants s2 and $s 5$ is reflected in the difference between the heterozygote macronuclei derived from each of them: For both alleles, the telomeric distribution of the heterozygote progeny of mutant $\mathrm{s} 2$ is farther downstream than that of mutant s5, just as the telomeric distribution of mutant $\mathrm{s} 2$ is farther downstream than that of mutant s5. Second, in a given heterozygote macronucleus the telomeric distribution is not the same for both alleles. It is always, on average, farther downstream for the 168 allele than for the 156 allele. On the blot shown in Figure 8A, the telomeric fragments of the 156 allele are less intense in the mutant $\mathrm{s} 5$ and the heterozygotes derived from this mutant than in the heterozygotes derived from the 168 parent. This is because the 156-specific probe, pPA2, corresponds to a sequence which is tandemly repeated five times in the coding sequence of the G gene. The telomere addition region of mutant 55 coincides with this repeated structure, so that only the longest of the telomeric fragments of this mutant can be labeled as intensely as the wild-type telomeric fragments. To quantify the number of copies of each allele in the heterozygotes, DNA was digested with $B a m H I$ and $H p h \mathbf{I}$, which gives a BamHI-HphI fragment (downstream of the BamHI site, but $5 \mathrm{~kb}$ upstream of the telomere addition region of mutant s5) of $880 \mathrm{bp}$ for the 156 allele and 692 bp for the 168 allele. The Southern blot shown in Figure $8 \mathrm{C}$ was probed with the corresponding part of fragment pEBGl and shows that the number of copies of each allele in the four caryonides of both crosses is roughly half that of the corresponding allele in the parents. Note that the probe used was derived from the 156 allele and does not hybridize as well with the 692 -bp fragment of the 168 allele.

\section{Discussion}

In this paper it was shown that transformation of $P$. primaurelia with a high copy number of a plasmid containing the $G$ gene specifically modifies the position at which telomeres are formed during the following macronuclear differentiation. The alternative telomere position results in the almost complete elimination of the $G$ gene from the new macronucleus, producing mutant progeny unable to express the G serotype. It is striking that the mutant telomere position is immediately upstream of the sequence contained in plasmid pX $\Delta 4$ in both 168 and 156 mutants, although this implies deletions of different lengths from the two wild-type telomeres. Whether this is a coincidence or a consequence of the mechanisms involved remains to be determined. Experiments are in progress to determine whether injection of plasmids carrying different inserts can induce dele- 


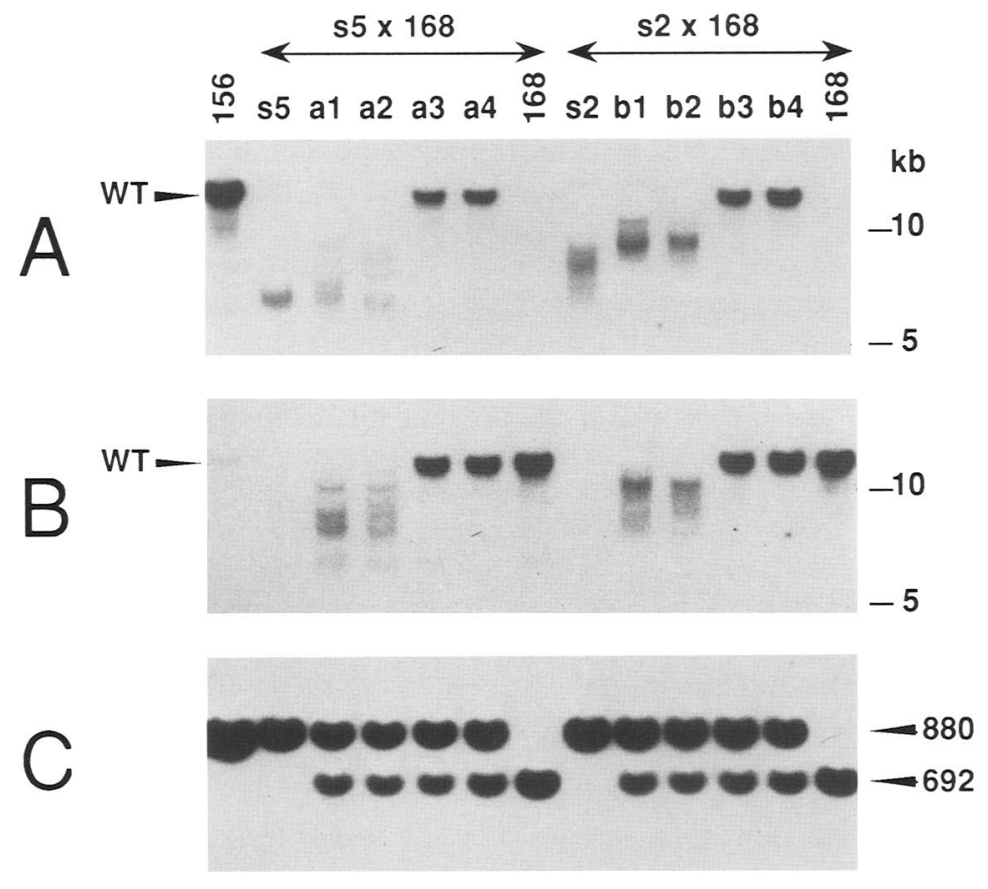

Figure 8. Molecular analysis of the crosses of $156 \mathrm{mu}$ tants s5 and s2 with the wild-type 168 straln. $(A)$ Southern blot of a $0.6 \%$ agarose gel electrophoresis of BamHI-digested DNA from the two parent clones and the four caryonides of each cross, probed with the 156specific probe pPA2. (Lanes $a 1, a 2)$ Caryonides derived from the mutant s5 parent; (lanes $a 3, a 4$ ) caryonides derived from the wild-type 168 parent; (lanes b1,b2) caryonides derived from the mutant $\mathrm{s} 2$ parent; (lanes $b 3, b 4)$ caryonides derived from the wild-type 168 parent; (lane 156) wild-type 156 DNA (this lane inadvertently contains twice as much DNA as the others). The arrowhead points to the wild-type telomeric fragment (WT). (B) Same blot as in $A$, dehybridized and rehybridized with the 168-specific probe pP11. (C) Southern blot of a $1 \%$ agarose gel electrophoresis of $\mathrm{BamHI}-$ and $H p h I$-digested DNA from the same clones, probed with a 467-bp fragment located $208-675$ bp downstream of the BamHI site of the 156 allele. The 156 lane contains twice as much DNA as the others. Arrowheads point to the position of the 880- and 692-bp BamHI-HphI fragments, respectively, derived from the 156 and the 168 alleles.

tions at other points of the sequence. The mutant telomere position is not stable through a sccond autogamy: Telomeres now form at a variety of different positions in different caryonides. However, the mutant telomere still exerts a strong influence on the processing of the micronuclear copy of the G gene, because few telomeres, if any, are formed at the wild-type position in each caryonide. The telomere position can be stabilized after a third autogamy, as shown by the mass autogamy experiment, in which the pattern obtained from a mass cultivation of 100 independent autogamous cells is similar to that of the parent. Crosses of the stabilized mutants with wild-type cells show that their micronuclei contain copies of the $G$ gene that can still be normally processed when the new macronucleus develops in a wild-type cell. Conversely, the micronuclear copies of wild-type cells are not normally processed in the mutant cells. Therefore, those mutations show the same macronuclear pattern of inheritance as $\mathrm{d} 48$, a previously described deletion mutant of $P$. tetraurelia (Epstein and Forney 1984), and confirm that this pattern of inheritance results from the influence of the old macronucleus on the process of developmental reorganization of the genome.

\section{Influence of the macronucleus on the processing of the macronuclear genome}

Although the data presented in this report are not sufficient to propose a general model explaining how the processing of micronuclear genes is affected by the content of the old macronucleus, some important points can be made. The wild-type 156 and s mutant strains have the same micronuclear genome, yet telomeres reproducibly form at different positions of the $G$ gene sequence at each autogamy. Whether or not the $G$ gene macronuclear telomere is produced by a breakage of the micronuclear chromosome during macronuclear development, the different telomere positions in the two strains cannot be determined by a cis-acting element such as the chromosome breakage sequence (Cbs) recently identified in Tetrahymena (Yao et al. 1990). Clearly, in this case, another mechanism is involved in the positioning of the telomeres, which is determined by the old macronucleus rather than by the micronuclear sequence. Therefore, a specific signal has to be transmitted from the old macronucleus to the developing one. This conclusion had already been reached in the study of the $\mathrm{d} 48$ mutation after it was shown that transplantation of macronucleoplasm from wild-type cells into the macronucleus of d48 allowed the mutant cells to revert permanently to wild type after the following autogamy (Harumoto 1986). It was further shown that microinjection of a plasmid containing the A gene into the macronucleus had the same effect; transfer of cytoplasm from wild-type cells to $\mathrm{d} 48$ cells also rescued the $\mathrm{d} 48$ mutation if donor and recipient cells were at specific stages in autogamy (Koizumi and Kobayashi 1989). Rescuing plasmids do not necessarily have to contain the whole A gene sequence: A plasmid lacking the last $1 \mathrm{~kb}$ of the coding sequence was able to rescue $\mathrm{d} 48$; however a plasmid containing only $2.1 \mathrm{~kb}$ from the middle of the coding sequence did not have this ability (You et al. 1991). These experiments have led to the hypothesis that a specific factor is produced by a defined region of the macronuclear $A$ gene and acts via the cytoplasm on the developing macronucleus to ensure normal processing of the A gene. 


\section{Evidence for mutant processing factors}

However, the question of what determines the position of the telomeres in the mutant macronucleus has not been addressed. The simplest explanation for the stable differences in telomere position between wild-type and mutant cell lines, both in $P$. tetraurelia and in $P$. primaurelia, is that the telomeres are positioned in the developing macronucleus just as they are in the old macronucleus. I propose that each macronuclear telomeric region is able to produce a specific factor that directs the processing of the corresponding micronuclear sequence by transmitting information about the position of telomeres to the developing macronucleus. Several lines of evidence support this idea. (1) The fact that wild-type $P$. primaurelia cells transformed with $\mathrm{pX} \Delta 4$ (but not uninjected controls nor cells transformed with a different replicating plasmid) produce progeny carrying a specific macronuclear deletion of the $G$ gene suggests that a specific factor produced from the telomerized plasmid is responsible for this deletion. In contrast to the $\mathrm{d} 48$ rescue experiments, the specific processing factor is needed here to explain formation of the telomeres at a mutant position. This factor could override the effect of the factor normally produced from the wild-type telomere, still present in normal amounts in the macronucleus of injected cells before autogamy, because of the high copy number of the plasmid. Indeed, in the experiment shown in Figure 2, the ratio of telomerized plasmid to wild-type telomeres before autogamy seems to be correlated with the ratio of mutant to wild-type telomeres after autogamy. (2) In the course of this study, many different telomere positions have been observed along the $\mathrm{G}$ genecoding and flanking sequences, which is consistent with previous observations that there appears to be no specific sequence requirement for the formation of telomeres. It was not proven that all telomere positions can be stable; however, the two crosses presented in Figure 8 show that mutants s2 and s5, which differ only slightly in the position of the telomeres, influence the processing of the micronuclear $168 \mathrm{G}$ gene differently, the telomere position in the mutant parent defining the upstream-most telomere position in the heterozygotes. Thus, these two mutants produce different specific processing factors.

\section{Stability of the macronuclear telomeric distribution}

What, then, would determine the stability of any particular macronuclear telomeric distribution through autogamy? It was shown that the outcome of the autogamy of the first generation mutants (those obtained after the autogamy of the injected cell lines) was variable (Fig. 5). Autogamy of the second generation mutant e5 also altered the telomeric distribution (Fig. 6). Although this does not seem to support the idea that telomeres always reproduce themselves at the same position, it should be noted that in both cases the telomere position was not unique in the macronucleus of the cells that underwent autogamy (first-generation mutants still retain some wild-type telomeres, while mutant e5 has a bimodal telomeric distribution). The heterogeneity in the postautogamous telomeric distributions could be linked to that preautogamous condition, if we assume that only a random subset of the processing factors produced by a macronucleus with a heterogeneous telomeric distribution is used for the determination of the new macronucleus. In addition, different processing factors might have different efficiencies that would result in different telomere positions having different stabilities, leading to unpredictable competition. The stabilization of the mutant telomere position after several successive rounds of autogamy could thus simply be due to the random selection of a particular telomere position. Under this hypothesis, the high frequency of spontaneous reversion of the d48 mutation at autogamy (You et al. 1991) could be linked to the reported presence of a small number of wild-type telomeres in the $\mathrm{d} 48$ macronucleus. It should also be noted that even when autogamy alters the pattern of telomeric distribution, a faint image of the old macronucleus distribution can still be seen in the new one (see Figs. 3C, 5, and 6), so that even unstable telomere positions seem to be able to reproduce in the new macronucleus. However, the appearance of telomere positions intermediate between the mutant and the wildtype positions after the autogamy of the first-generation mutants remains difficult to explain.

\section{Predetermination of the micronuclei by the processing factors?}

The crosses of the stabilized 156 mutants with strain 168 show that the telomeric distributions of both alleles are heterogeneous in the heterozygote caryonides derived from the mutant parent. This appears to be at odds with the fact that autogamy of the 156 mutants introduces little or no heterogeneity in the telomeric distribution. How could the mutant macronucleus affect differently the processing of the 156 allele in the homozygous nuclei of autogamous mutant cells and in the heterozygous nuclei of the mutant exconjugants? One possibility is that the haploid pronuclei are at least partially predetermined by the processing factors even before they are exchanged between the mating cells. This is consistent with the timing of production of the processing factors determined by the cytoplasm transfer experiments of Koizumi and Kobayashi (1989). They showed that the ability of cytoplasm from wild-type autogamous cells to rescue the $\mathrm{d} 48$ mutation could already be detected at the stage of lobed macronucleus, well before the stage corresponding to the exchange of the pronuclei in conjugation. Processing of the genes in the developing heterozygote macronuclei of the mutant exconjugant would then introduce the same heterogeneity as is seen in the macronuclei developing in an autogamous cell where the old macronucleus shows a heterogeneous telomeric distribution. The fact that the telomeric distribution of the 168 alleles in these heterozygote macronuclei is different from that of the 156 alleles, and closer to the wild-type, may be related to the predetermination of micronuclear copies. Perhaps this could also explain the occasional 
formation of wild-type telomeres in the caryonides derived from a $\mathrm{d} 48$ cell crossed with a wild-type cell. The asymmetry of these crosses (macronuclear development on the wild-type side does not seem to be influenced by the micronucleus coming from the mutant cell) could mean that the processing factors corresponding to the wild-type telomere position have a dominant effect, or are more efficient than others, giving a greater stability to wild-type telomeres. Interestingly, an asymmetrical predetermination of the micronuclei had already been postulated to explain deviations from the normally macronuclear inheritance of the mating type differentiation in P. tetraurelia (Brygoo et al. 1980).

If indeed each macronuclear telomeric region is able to produce a specific factor, and since there appears to be no stringent sequence requirement for the formation of macronuclear telomeres, it is difficult to escape the conclusion that the factors are the telomeric regions themselves, or a copy of them. Whereas the molecular nature of the processing factors remains unknown, a careful study of what is required for the injected plasmid to induce deletions (copy number, form, sequence, patterns of linearization, and telomerization/ may help define their role in the processing of the micronuclear genes. Whatever this role may be, it is now possible to induce experimentally specific modifications of the telomerization pattern during macronuclear differentiation.

\section{Materials and methods}

Cell lines and cultivation

P. primaurelia wild-type strains 156 and 168 (Sonneborn 1974) are well-characterized stocks that have been used extensively in genetic studies of the surface antigen system. Cells were grown in a grass infusion medium bacterized the day before use with Klebsiella pneumoniae and supplemented with $0.8 \mathrm{mg} / \mathrm{liter}$ of $\beta$-sitosterol (Merck, Darmstadt, Germany). Cultivation, autogamy, and conjugation were all carried out at $24^{\circ} \mathrm{C}$. Basic methods of cell culture have been described (Sonneborn 1970).

\section{Injected DNA}

Plasmid pX $\Delta 4$ is a derivative of pXI3, a larger plasmid containing the G gene from strain 156 (Caron and Meyer 1985), and contains the entire transcribed region $(8218 \mathrm{bp})$ as well as $230 \mathrm{bp}$ of upstream and about 510 bp of downstream flanking sequences. The sequence of the whole insert was determined and partly published (Prat et al. 1986).

\section{Autogamy and conjugation}

Autogamy was induced by starving the cells after they had reached the appropriate clonal age, and assessed by staining with a $15: 1(\mathrm{vol} / \mathrm{vol})$ mixture of carmine red $10.5 \%$ in $45 \%$ acetic acid) and fast green (1\% in ethanol). Cells were isolated from depressions showing $>50 \%$ autogamous cells; autogamy was checked by staining three cells out of four after the second division of the isolated cell to verify the presence of macronuclear fragments. Conjugation was induced by starving two clones with complementary mating types. The four caryonides from individual pairs were isolated; the cytoplasmic origin of each of the caryonides was determined by an early immobiliza- tion test after two more divisions, and further confirmed after DNA extraction by the identification of the mitochondrial DNA restriction pattern, which is strain specific.

\section{Microinjections}

Caryonidal clones of 15-30 fissions of age were injected in Dryl's medium (Dryl 1959) containing 0.2\% BSA, under an oil film, while being visualized with a phase-contrast inverted microscope (Axiovert 35M, Zeiss). Approximately $5 \mathrm{pl}$ of a solution of $\mathrm{CsCl}$-purified plasmid DNA, that had been filtered on a $0.22-\mu \mathrm{m}$ Millex-GV4 filter (Millipore) and disolved in water at a concentration of $5 \mathrm{mg} / \mathrm{ml}$, was delivered into the macronucleus.

\section{DNA extraction}

Cultures $(400 \mathrm{ml})$ of exponentially growing cells at 1000 cells/ $\mathrm{ml}$ were centrifuged. After being washed in Dryl's medium, the pellet was resuspended in one volume of the same buffer, and quickly added to four volumes of lysis solution $[0.44 \mathrm{M}$ EDTA (pH 9.0), 1\% SDS, $0.5 \% N$-laurylsarcosine (Sigma), and $1 \mathrm{mg} / \mathrm{ml}$ proteinase $\mathrm{K}$ (Merck) $)$ at $55^{\circ} \mathrm{C}$. The lysate was incubated at $55^{\circ} \mathrm{C}$ for at least $5 \mathrm{hr}$, gently extracted once with phenol, and dialyzed twice against TE [10 mM Tris- $\mathrm{HCl}, 1 \mathrm{~mm}$ EDTA [pH 8.0)] containing $20 \%$ ethanol, and once against TE.

\section{DNA restriction and electrophoresis}

These procedures were carried out according to standard methods (Sambrook et al. 1989). Pulse-field electrophoresis was carried out in a contour-clamped homogeneous electric field (CHEF) home-made apparatus (Chu et al. 1986) in $0.25 \times$ TBE (TBE is $89 \mathrm{~mm}$ Tris, $89 \mathrm{~mm}$ borate, and $2.5 \mathrm{~mm}$ EDTA) at 5 $\mathrm{V} / \mathrm{cm}$, with cooling to $8^{\circ} \mathrm{C}$ and a commutation period of $4 \mathrm{sec}$.

\section{Southern hybridization}

DNA was transferred from agarose gels to Hybond $\mathrm{N}^{+}$membranes (Amersham, $\mathrm{UK}$ ) in $0.4 \mathrm{~N} \mathrm{NaOH}$ after depurination in $0.25 \mathrm{~N} \mathrm{HCl}$. Hybridization was carried out according to Church and Gilbert (1984) in 7\% SDS, $0.5 \mathrm{M}$ sodium phosphate, $1 \%$ $\mathrm{BSA}$, and $1 \mathrm{mM}$ EDTA $(\mathrm{pH} 7.2)$ at $63^{\circ} \mathrm{C}$. Probes were labeled using a random priming kit (Boehringer Mannheim) to a specific activity of $3 \times 10^{9} \mathrm{cpm} / \mu \mathrm{g}$. Membranes were then washed for $30 \mathrm{~min}$ in $0.2 \times \mathrm{SSC}$ /SSC is $0.15 \mathrm{M} \mathrm{NaCl}$ and $0.015 \mathrm{M}$ sodium citrate) and $0.5 \%$ SDS at $63^{\circ} \mathrm{C}$ (allele-specific probes pPA2 and $\mathrm{pP} 11$ ) or $60^{\circ} \mathrm{C}$ (other probes) prior to autoradiography.

\section{Acknowledgments}

I thank François Caron for the gift of probe LKlA; Alain Butler, François Caron, Michaël D. Katinka, and Linda D. Martin for critically reading the manuscript; Anne-Marie Keller and Anne Le Mouël for sharing unpublished data; and all of the above for stimulating discussions. Plasmid YEpJB1-23-0 was kindly given by Agnès Delahodde. This work was supported by grant $88 / 1470$ from the Direction des Recherches, Etudes et Techniques, Ministère de la Défense, and by a grant from the Ministère de la Recherche et de l'Education Supérieure (Structure et Fonction des Macromolcules et Systèmes Intégrés).

The publication costs of this article were defrayed in part by payment of page charges. This article must therefore be hereby marked "advertisement" in accordance with 18 USC section 1734 solely to indicate this fact. 


\section{References}

Allen, S. and I. Gibson. 1972. Genome amplification and gene expression in the ciliate macronucleus. Biochem. Genet. 6: $293-313$.

Banroques, J., J. Perea, and C. Jacq. 1987. Efficient splicing of two yeast mitochondrial introns controlled by a nuclear-encoded maturase. EMBO I. 6: 1085-1091.

Baroin, A., A. Prat, and F. Caron. 1987. Telomeric site position heterogeneity in macronuclear DNA of Paramecium primaurelia. Nucleic Acids Res. 15: 1717-1728.

Beale, G.H. 1952. Antigen variation in Paramecium aurelia, variety 1. Genetics 37: 62-74.

Brygoo, Y., T.M. Sonneborn, A.-M. Keller, R.V. Dippel, and M.V. Schneller. 1980. Genetic analysis of mating type differentiation in Paramecium tetraurelia. II. Role of the micronuclei in mating type differentiation. Genetics 94: 951-959.

Caron, F. and E. Meyer. 1985. Does Paramecium primaurelia use a different genetic code in its macronucleus? Nature 314: $185-188$.

- 1989. Molecular basis of surface antigen variation in paramecia. Annu. Rev. Microbiol. 43: 23-42.

Chu, G., D. Vollrath, and R.W. Davis. 1986. Separation of large DNA molecules by contour-clamped homogenous electric fields. Science 234: 1582-1585.

Church, G.M. and W. Gilbert. 1984. Genomic sequencing. Proc. Natl. Acad. Sci. 81: 1991-1995.

Cummings, D.J. 1975. Studies on macronuclear DNA from Paramecium aurelia. Chromosoma 53: 191-208.

Dryl, S. 1959. Antigenic transformation in Paramecium aurelia after homologous antiserum treatment during autogamy and conjugation. /. Protozool. (Suppl.) 6: 25.

Epstein, L.M. and J.D. Forney. 1984. Mendelian and non-Mendelian mutations affecting surface antigen expression in Paramecium tetraurelia. Mol. Cell. Biol. 4: 1583-1590.

Forney, J.D. and E.H. Blackburn. 1988. Developmentally controlled telomere addition in wild-type and mutant paramecia. Mol. Cell. Biol. 8: 251-258.

Gibson, I. and N. Martin. 1971. DNA amounts in the nuclei of Paramecium aurelia and Tetrahymena pyriformis. Chromosoma 35: 374-382.

Gilley, D., J.R. Preer, Jr., K.J. Aufderheide, and B. Polisky. 1988. Autonomous replication and addition of telomerelike sequences to DNA microinjected into Paramecium tetraurelia macronuclei. Mol. Cell. Biol. 8: 4765-4772.

Harumoto, T. 1986. Induced change in a non-Mendelian determinant by transplantation of macronucleoplasm in Paramecium tetraurelia. Mol. Cell. Biol. 6: 3498-3501.

Katinka, M.D. and F.M. Bourgain. 1992. Interstitial telomeres are hotspots for illegitimate recombination with DNA molecules injected into the macronucleus of Paramecium primaurelia. $E M B O$ J. 11: (in press).

Koizumi, S. and S. Kobayashi. 1989. Microinjection of plasmid DNA encoding the A surface antigen of Paramecium tetraurelia restores the ability to regenerate a wild-type macronucleus. Mol. Cell. Biol. 9: 4398-4401.

McTavish, C. and J. Sommerville. 1980. Macronuclear DNA organization and transcription in Paramecium primaurelia. Chromosoma 78: 147-164.

Prat, A. 1990. Conserved sequences flank variable tandem repeats in two alleles of the G surface protein of Paramecium primaurelia. J. Mol. Biol. 211: 521-535.

Prat, A., M. Katinka, F. Caron, and E. Meyer. 1986. Nucleotide sequence of the Paramecium primaurelia $\mathrm{G}$ surface protein. A huge protein with a highly periodic structure. I. Mol. Biol. 189: $47-60$.
Preer, J.R., Jr. and L.B. Preer. 1979. The size of macronuclear DNA and its relationship to models for maintaining genic balance. I. Protozool. 26: 14-18.

Sambrook, J., E.F. Fritch, and T. Maniatis. 1989. Molecular cloning: A laboratory manual, 2nd ed. Cold Spring Laboratory Press, Cold Spring Harbor, New York.

Sonneborn, T.M. 1970. Methods in Paramecium research. Methods Cell Physiol. 4: 241-339.

1974. Paramecium aurelia. In Handbook of genetics: Plants, plant viruses and protists (ed R.C. King), vol. 2, pp. 469-594. Plenum Press, New York.

1977. Genetics of cellular differentiation: Stable nuclear differentiation in eucaryotic unicells. Annu. Rev. Genet. 11: 349-367.

Sonneborn, T.M. and M.V. Schneller. 1979. A genetic system for alternative stable characteristics in genomically identical homozygous clones. Dev. Genet. 1: 21-46.

Yao, M.-C. 1989. Site-specific chromosome breakage and DNA deletion in ciliates. In Mobile DNA. American Society for Microbiology, Washington, D.C.

Yao, M.-C., C.-H. Yao, and B. Monks. 1990. The controlling sequence for site-specific chromosome brekage in Tetrahymena. Cell 63: 763-772.

You, Y., K. Aufderheide, J. Morand, K. Rodkey, and J. Forney. 1991. Macronuclear transformation with specific DNA fragments controls the content of the new macronuclear genome in Paramecium tetraurelia. Mol. Cell. Biol. 11: 11331137. 


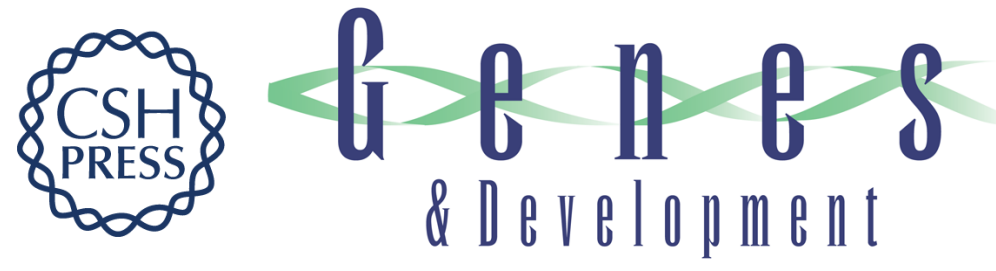

\section{Induction of specific macronuclear developmental mutations by microinjection of a cloned telomeric gene in Paramecium primaurelia.}

E Meyer

Genes Dev. 1992, 6:

Access the most recent version at doi:10.1101/gad.6.2.211

References This article cites 26 articles, 9 of which can be accessed free at: http://genesdev.cshlp.org/content/6/2/211.full.html\#ref-list-1

License

Email Alerting

Service

Receive free email alerts when new articles cite this article - sign up in the box at the top right corner of the article or click here.

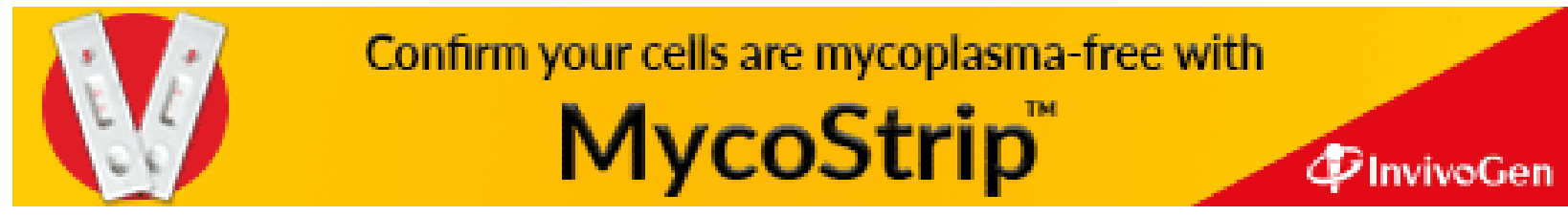

